\title{
Nontrivial order preserving automorphisms of non-Archimedean fields
}

\author{
Khodr Shamseddine
}

\begin{abstract}
A study of order preserving field automorphisms of ordered nonArchimedean field extensions of $\mathbb{R}$ will be presented. We show that, while the identity map is the only field automorphism of $\mathbb{R}$, infinitely many nontrivial order preserving field automorphisms can be constructed on an ordered nonArchimedean field extension $F$ of $\mathbb{R}$. Moreover, we show that if $P$ is an order preserving field automorphism of $F$ then $P(r) \approx r$ for all $r \neq 0$ in $\mathbb{R} \subset$ $F$; and invoking the axiom of choice, we construct an order preserving field automorphism $P$ of $F$ satisfying $P(r) \neq r$ for infinitely many $r \in \mathbb{R}$.
\end{abstract}

\section{Introduction}

We start this section by reviewing some basic terminology and facts about nonArchimedean fields. So let $F$ be an ordered non-Archimedean field extension of $\mathbb{R}$. We introduce the following terminology.

Definition $1.1\left(\sim, \approx, \ll, S_{F}, \lambda\right)$. For $x, y \in F^{*}:=F \backslash\{0\}$, we say $x \sim y$ if there exist $n, m \in \mathbb{N}$ such that $n|x|>|y|$ and $m|y|>|x|$, where $|\cdot|$ denotes the usual absolute value on $F$ :

$$
|x|= \begin{cases}x & \text { if } x \geq 0 \\ -x & \text { if } x<0 .\end{cases}
$$

For nonnegative $x, y \in F$, we say that $x$ is infinitely smaller than $y$ and write $x \ll y$ if $n x<y$ for all $n \in \mathbb{N}$, and we say that $x$ is infinitely small if $x \ll 1$ and $x$ is finite if $x \sim 1$; finally, we say that $x$ is approximately equal to $y$ and write $x \approx y$ if $x \sim y$ and $|x-y| \ll|x|$. We also set $\lambda(x)=[x]$, the class of $x$ under the equivalence relation $\sim$.

The set of equivalence classes $S_{F}$ (under the relation $\sim$ ) is naturally endowed with an addition via $[x]+[y]=[x \cdot y]$ and an order via $[x]<[y]$ if $|y| \ll|x|$ (or $|x| \gg|y|$ ), both of which are readily checked to be well-defined. It follows that $\left(S_{F},+, \leq\right)$ is an ordered group, often referred to as the Hahn group or skeleton group, whose neutral element is [1], the class of 1 . It follows from the above that

2000 Mathematics Subject Classification. 08A35, 26E30, 12J25, 11D88.

Key words and phrases. non-Archimedean field, skeleton group, field automorphism.

(C)2011 American Mathematical Society 
the projection $\lambda$ from $F^{*}$ to $S_{F}$ is a valuation. Throughout this paper, the ordered group $\left(S_{F},+, \leq\right)$ will be simply denoted by $S_{F}$.

The theorem of Hahn [2] provides a complete classification of non-Archimedean extensions of $\mathbb{R}$ in terms of their skeleton groups. In fact, invoking the axiom of choice it is shown that the elements of any such field $F$ can be written as formal power series over its skeleton group $S_{F}$ with real coefficients, and the set of appearing exponents forms a well-ordered subset of $S_{F}$.

From general properties of formal power series fields $[\mathbf{6}, \mathbf{8}]$, it follows that if $S_{F}$ is divisible then $F$ is real closed; that is, every positive element of $F$ is a square in $F$ and every polynomial of odd degree over $F$ has at least one root in $F$. For a general overview of the algebraic properties of formal power series fields, we refer to the comprehensive overview by Ribenboim [9], and for an overview of the related valuation theory the book by Krull [3]. A thorough and complete treatment of ordered structures can also be found in [7].

Our research $[12,10,13,14,20,15,16,21,17,18,19,11]$ has been focused on the study of any ordered non-Archimedean field extension of $\mathbb{R}$ that is complete in the order topology and whose skeleton group is Archimedean, i.e. a subgroup of $\mathbb{R}$. The smallest such field is the field $L$ of the formal Laurent series whose skeleton group is $\mathbb{Z}$; and the smallest such field that is also real closed is the Levi-Civita field $\mathcal{R}$, first introduced in $[4,5]$. In this case, $S_{\mathcal{R}}=\mathbb{Q}$. We recall here that the elements of $\mathcal{R}$ are functions from $\mathbb{Q}$ to $\mathbb{R}$ with left-finite supports, i.e. below any rational bound $r$ there are only finitely many points where the function does not vanish; given an element $x \in \mathcal{R}$ and $q \in \mathbb{Q}$, then the value of $x$ (as a function from $\mathbb{Q}$ to $\mathbb{R}$ ) at $q$ is denoted by $x[q]$. The infinitely small and positive number $d$ is defined by $d[1]=1$ and $d[q]=0$ for $q \neq 1$. For all $x \in \mathcal{R}$, the elements of the support of $x$ can be arranged in ascending order, say $\operatorname{supp}(x)=\left\{q_{1}, q_{2}, \ldots\right\}$ with $q_{j}<q_{j+1}$ for all $j$; and $x$ can be written as $x=\sum_{j=1}^{\infty} x\left[q_{j}\right] d^{q_{j}}$, where the series converges in the topology induced by the order $[\mathbf{1}]$ and where for $x \neq 0$

$$
x>0 \text { if } x\left[q_{1}\right]>0 \text { and } x<0 \text { if } x\left[q_{1}\right]<0 .
$$

The Levi-Civita field $\mathcal{R}$ is of particular interest because of its practical usefulness. Since the supports of the elements of $\mathcal{R}$ are left-finite, it is possible to represent these numbers on a computer [1]. Having infinitely small numbers, the errors in classical numerical methods can be made infinitely small and hence irrelevant in all practical applications. One such application is the computation of derivatives of real functions representable on a computer [12], where both the accuracy of formula manipulators and the speed of classical numerical methods are achieved.

In the next two sections, we prove general results about skeleton groups and field automorphisms which will be useful for understanding the structure of nonArchimedean fields. We show that if $F$ is an ordered non-Archimedean field extension of $\mathbb{R}$ and if $P$ is an order preserving filed automorphism of $F$ then $P$ induces an order preserving group automorphism on $S_{F}$, the skeleton group of $F$. We also show that we can construct infinitely many nontrivial order preserving field automorphisms of $F$, all of which have to satisfy the property $P(r) \approx r$ for all $r \neq 0$ in $\mathbb{R} \subset F$; moreover, an order preserving field automorphism $P$ can be defined on $F$ so that $P(r) \neq r$ for uncountably many $r \in \mathbb{R}$. 


\section{Skeleton Groups}

In this section we classify ordered fields based on the size and the properties of their skeleton groups.

Definition 2.1. Let $F$ be an ordered field. Then we say that $F$ is nonArchimedean if the skeleton group $S_{F}$ of $F$ contains more than one element.

As mentioned in the introduction, the skeleton groups of $L$ and $\mathcal{R}$ are, respectively, $S_{L}=\mathbb{Z}$ and $S_{\mathcal{R}}=\mathbb{Q}$; and hence $L$ and $\mathcal{R}$ are non-Archimedean. However, $\mathbb{R}$ is not non-Archimedean (it is Archimedean) since its skeleton group is $S_{\mathbb{R}}=\{0\}=\{[1]\}$; and this is of course one of the defining properties of $\mathbb{R}$.

Proposition 2.2. Let $F$ be an ordered non-Archimedean field. Then $\mathbb{Z} \subset S_{F}$.

Proof. Since $F$ is non-Archimedean, there exists an element $d \in F^{*}$ such that $[d] \neq 0=[1]$. Since $[d]=[-d]$, we may assume that $d>0$. Since $-[d]=\left[d^{-1}\right] \in S_{F}$, we may assume that $[d]>0$, i.e. that $d$ is infinitely small. Consider the subset $Z_{F}=\left\{\left[d^{n}\right]: n \in \mathbb{Z}\right\}$ of $S_{F}$. For $m>n$, we have that

$$
\begin{aligned}
-\left[d^{n}\right]+\left[d^{m}\right] & =\left[d^{-n} \cdot d^{m}\right]=\left[d^{m-n}\right]=[\underbrace{d \cdot d \cdot \ldots \cdot d}_{(m-n) \text { times }}] \\
& =\underbrace{[d]+[d]+\cdots+[d]}_{(m-n) \text { times }} \\
& >0 .
\end{aligned}
$$

Thus, $m \neq n \Rightarrow\left[d^{m}\right] \neq\left[d^{n}\right]$ for all $m, n \in \mathbb{Z}$. The map $P: Z_{F} \rightarrow \mathbb{Z}$ given by

$$
P\left(\left[d^{n}\right]\right)=n
$$

is an order preserving isomorphism; that is,

(1) $\mathrm{P}$ is a bijection,

(2) for all $m, n \in \mathbb{Z}$, we have that

$$
P\left(\left[d^{m}\right]+\left[d^{n}\right]\right)=P\left(\left[d^{m}\right]\right)+P\left(\left[d^{n}\right]\right),
$$

and

(3) for all $m, n \in \mathbb{Z}$,

$$
\left[d^{m}\right]<\left[d^{n}\right] \Leftrightarrow P\left(\left[d^{m}\right]\right)<P\left(\left[d^{n}\right]\right) .
$$

Thus, $\mathbb{Z}$ is isomorphic to a subset of $S_{F}$, or simply $\mathbb{Z} \subset S_{F}$.

Proposition 2.3. Let $F$ be an ordered non-Archimedean field which admits roots of positive elements. Then $\mathbb{Q} \subset S_{F}$.

Proof. Let $d \in F^{*}$ be as in the proof of Proposition 2.2. Let $q>0$ in $\mathbb{Q}$ be given; write $q=m / n$ where $m, n \in \mathbb{N}$. Thus, $\left[d^{m}\right]>0$. Using the fact that $\left[d^{m}\right]=\left[d^{n \cdot q}\right]=\underbrace{\left[d^{q}\right]+\left[d^{q}\right]+\cdots+\left[d^{q}\right]}_{n \text { times }}$, we obtain that $\left[d^{q}\right]>0$. In particular, $\left[d^{q}\right] \neq 0$.

Now let $q_{1} \neq q_{2}$ be given in $\mathbb{Q}$. We may assume that $q_{2}>q_{1}$. Then $q_{2}-q_{1}>0$, and hence

$$
-\left[d^{q_{1}}\right]+\left[d^{q_{2}}\right]=\left[d^{-q_{1}}\right]+\left[d^{q_{2}}\right]=\left[d^{q_{2}-q_{1}}\right]>0 .
$$

Thus, $q_{1} \neq q_{2} \Rightarrow\left[d^{q_{1}}\right] \neq\left[d^{q_{2}}\right]$. 
Let $Q_{F}=\left\{\left[d^{q}\right]: q \in \mathbb{Q}\right\}$. Then $Q_{F}$ is a subgroup of $S_{F}$, and the map $P$ : $Q_{F} \rightarrow \mathbb{Q}$, given by

$$
P\left(\left[d^{q}\right]\right)=q,
$$

is an order preserving group isomorphism from $Q_{F}$ onto $\mathbb{Q}$.

REMARK 2.4. Let $F$ and $Q_{F}$ be as in Proposition 2.3. If we define $\times: Q_{F} \times$ $Q_{F} \rightarrow Q_{F}$ by

$$
\left[d^{q_{1}}\right] \times\left[d^{q_{2}}\right]=\left[d^{q_{1} \cdot q_{2}}\right]
$$

then $\left(Q_{F},+, \times, \leq\right)$ is an ordered field, and the map $P$ given in Equation (2.1) is a field isomorphism of $Q_{F}$ onto $\mathbb{Q}$.

\section{Field Automorphisms}

Definition 3.1. Let $F$ be a field. Then we say that $P$ is an automorphism of $F$ if $P$ is an isomorphism from $F$ onto itself.

Lemma 3.2. Let $S$ and $T$ be fields, and let $P: S \rightarrow T$ be a field isomorphism. Then $P$ has an inverse $P^{-1}: T \rightarrow S$ which is itself a field isomorphism from $T$ onto $S$.

Proof. Since $P$ is a bijection, $P^{-1}$ exists and it is a bijection. Let $+_{S}$ and $+_{T}$ denote the addition operations in $S$ and $T$, respectively; and let $\times_{S}$ and $\times_{T}$ denote the operations of multiplication in $S$ and $T$, respectively. Now let $y_{1}, y_{2} \in T$ be given, and let $x_{1}=P^{-1}\left(y_{1}\right)$ and $x_{2}=P^{-1}\left(y_{2}\right)$. Then

$$
\begin{aligned}
P^{-1}\left(y_{1}+_{T} y_{2}\right) & =P^{-1}\left(P\left(x_{1}\right)+_{T} P\left(x_{2}\right)\right) \\
& =P^{-1}\left(P\left(x_{1}+{ }_{S} x_{2}\right)\right) \text { since } P \text { is an isomorphism } \\
& =x_{1}+_{S} x_{2}=P^{-1}\left(y_{1}\right)+_{S} P^{-1}\left(y_{2}\right),
\end{aligned}
$$

and

$$
\begin{aligned}
P^{-1}\left(y_{1} \times_{T} y_{2}\right) & =P^{-1}\left(P\left(x_{1}\right) \times_{T} P\left(x_{2}\right)\right) \\
& =P^{-1}\left(P\left(x_{1} \times_{S} x_{2}\right)\right) \text { since } P \text { is an isomorphism } \\
& =x_{1} \times_{S} x_{2}=P^{-1}\left(y_{1}\right) \times_{S} P^{-1}\left(y_{2}\right) .
\end{aligned}
$$

Thus, $P^{-1}$ is a field isomorphism from $T$ onto $S$.

Proposition 3.3. Let $S$ and $T$ be ordered fields, and let $P: S \rightarrow T$ be an order preserving field isomorphism. Then $P^{-1}$ is an order preserving field isomorphism from $T$ onto $S$.

Proof. Using Lemma 3.2, it remains to show that $P^{-1}: T \rightarrow S$ is order preserving. Let $\leq_{S}$ and $\leq_{T}$ denote the order relations in $S$ and $T$, respectively; let $y_{1}, y_{2} \in T$ be such that $y_{1} \leq_{T} y_{2}$; and let $x_{1}=P^{-1}\left(y_{1}\right)$ and $x_{2}=P^{-1}\left(y_{2}\right)$. We need to show that $x_{1} \leq_{S} x_{2}$. Suppose not; then $x_{2}<_{S} x_{1}$. Since $P$ is order preserving, we obtain that $y_{2}=P\left(x_{2}\right)<_{T} P\left(x_{1}\right)=y_{1}$, a contradiction.

Lemma 3.4. Let $F$ be an ordered field, and let $P: F \rightarrow F$ be an automorphism of $F$. Then $P(q)=q$ for all $q \in \mathbb{Q}$. 
Proof. Since $F$ is an ordered field, $\mathbb{Q} \subset F$. For any $x \in F$, we have that $P(x)=P(0+x)=P(0)+P(x)$; and hence $P(0)=0$. Since $P$ is one-to-one, it follows that $P(x) \neq 0$ for all $x \neq 0$ in $F$. Thus, from $P(1)=P(1 \cdot 1)=P(1) \cdot P(1)$, we obtain that $P(1)=1$. Using induction and the fact that $P(l+1)=P(l)+P(1)=$ $P(l)+1$ for all $l \in \mathbb{N}$, we then readily obtain that $P(n)=n$ for all $n \in \mathbb{N}$.

Now let $q>0$ in $\mathbb{Q}$ be given; then $q=m / n$ for some $m, n \in \mathbb{N}$. Thus, $m=n \cdot q$. It follows that

$$
P(m)=P(n) \cdot P(q),
$$

from which we get that $m=n \cdot P(q)$. Hence $P(q)=m / n=q$.

Finally, let $q<0$ in $\mathbb{Q}$ be given; then $-q>0$. Thus, $P(-q)=-q$. Since $0=P(0)=P(-q+q)=P(-q)+P(q)$, it follows that $P(q)=-P(-q)=q$. Hence $P(q)=q$ for all $q \in \mathbb{Q}$.

Corollary 3.5. The identity map is the only field automorphism of $\mathbb{Q}$.

THEOREM 3.6. Let $F$ be an ordered Archimedean field. Then the identity map is the only order preserving field automorphism of $F$.

Proof. Assume not. Then there exists a nontrivial order preserving field automorphism $P$ of $F$. Thus, there exists $x \in F \backslash \mathbb{Q}$ such that $P(x) \neq x$. By Proposition 3.3, $P^{-1}$ is also an order preserving field automorphism of $F$, and $P^{-1}(x) \neq P^{-1}(P(x))=x$. If $P(x)<x$, then $x=P^{-1}(P(x))<P^{-1}(x)$. So we may assume without loss of generality that $x<P(x)$.

Since $F$ is an ordered Archimedean field, there exists $q \in \mathbb{Q} \subset F$ such that

$$
x<q<P(x) .
$$

Applying $P$ to the first inequality in (3.1) and using Lemma 3.4 and the fact $P$ is order preserving, we obtain that $P(x)<P(q)=q$, which contradicts the second inequality in (3.1). Hence the identity map is the only order preserving field automorphism of $F$.

LEMMA 3.7. Let $F$ be an ordered field which admits roots of positive elements, and let $P$ be a field automorphism of $F$. Then $P$ is order preserving.

Proof. It suffices to show that $P(a)>0$ for all $a>0$ in $F$; so let $a>0$ in $F$ be given. Let $b>0$ in $F$ be such that $b^{2}=a$. Hence

$$
P(a)=P\left(b^{2}\right)=(P(b))^{2} \geq 0 .
$$

Since $a \neq 0$ and since $P$ is one-to-one, we obtain that $P(a) \neq 0$. Thus, $P(a)>0$.

Combining the results of Theorem 3.6 and Lemma 3.7, we obtain the following result.

COROLlary 3.8. The identity map is the only field automorphism of $\mathbb{R}$.

Proposition 3.9. Let $F$ be an ordered non-Archimedean field, let $S_{F}$ be the skeleton group of $F$, and let $P$ be an order preserving field automorphism of $F$. Then the map $\Gamma: S_{F} \rightarrow S_{F}$, given by $\Gamma([x])=[P(x)]$, is a well-defined order preserving group automorphism of $S_{F}$.

Proof. Let $x \in F^{*}$ be given; then $P(x) \neq 0$. If $x<0$, then $P(x)<0$ and hence $P(|x|)=P(-x)=-P(x)=|P(x)|$. On the other hand, if $0<x$, then $0<P(x)$ and hence $P(|x|)=P(x)=|P(x)|$. So for all $x \in F^{*}, P(|x|)=|P(x)|$. 
To show that $\Gamma$ is a well-defined map, we need to show that

$$
[x]=[y] \Rightarrow[P(x)]=[P(y)] .
$$

So let $x, y \in F^{*}$ be such that $[x]=[y]$. Then there exist $m, n \in \mathbb{N}$ such that $|y|<m \cdot|x|$ and $|x|<n \cdot|y|$. Thus,

$$
|P(y)|=P(|y|)<P(m \cdot|x|)=P(m) \cdot P(|x|)=m \cdot|P(x)| ;
$$

and similarly, we show that $|P(x)|<n \cdot|P(y)|$. Hence $[P(x)]=[P(y)]$.

Now we show that $\Gamma$ is one to one. So let $[x],[y] \in S_{F}$ be such that $\Gamma([x])=$ $\Gamma([y])$. Since $[P(x)]=[P(y)]$, there exist $k, l \in \mathbb{N}$ such that $|P(y)|<k \cdot|P(x)|$ and $|P(x)|<l \cdot|P(y)|$. From $|P(y)|<k \cdot|P(x)|$ we obtain that

$$
P(|y|)<P(k) \cdot P(|x|)=P(k \cdot|x|) \text {; and hence }|y|<k \cdot|x| \text {. }
$$

Similarly, $|P(x)|<l \cdot|P(y)|$ entails that $|x|<l \cdot|y|$. Hence $[x]=[y]$.

To show that $\Gamma$ is onto, let $[y] \in S_{F}$ be given. We need to find $[x] \in S_{F}$ such that $[y]=\Gamma([x])$. Since $P$ is onto, there exists $x \in F$ such that $y=P(x)$. Since $y \neq 0$, we have also that $x \neq 0$. Hence $[x] \in S_{F}$ and $\Gamma([x])=[P(x)]=[y]$.

For any $[x],[y] \in S_{F}$, we have that

$$
\begin{aligned}
\Gamma([x]+[y]) & =\Gamma([x \cdot y])=[P(x \cdot y)]=[P(x) \cdot P(y)] \\
& =[P(x)]+[P(y)]=\Gamma([x])+\Gamma([y])
\end{aligned}
$$

It remains to show that $\Gamma$ preserves order in $S_{F}$. So let $[x],[y] \in S_{F}$ be such that $[x]<[y]$; then for all $n \in \mathbb{N}, n \cdot|y|<|x|$. It follows that

$$
n \cdot|P(y)|=P(n) \cdot P(|y|)=P(n \cdot|y|)<P(|x|)=|P(x)| \text { for all } n \in \mathbb{N} .
$$

Thus, $[P(x)]<[P(y)]$; and hence $\Gamma([x])<\Gamma([y])$.

Corollary 3.10. Let $F, P$, and $\Gamma$ be as in Proposition 3.9. Define $\Lambda: S_{F} \rightarrow$ $S_{F}$ by $\Lambda([x])=\left[P^{-1}(x)\right]$. Then $\Lambda$ is an order preserving group automorphism of $S_{F}$, and $\Lambda=\Gamma^{-1}$.

COROLlary 3.11. Let $F$ be an ordered non-Archimedean field extension of $\mathbb{R}$, and let $P$ be an order preserving field automorphism of $F$. Then, for all $r \in \mathbb{R}^{*}$, $P(r) \approx r$.

Proof. Let $r \in \mathbb{R}^{*}$ be given; then $[r]=[1]=0$. Hence, by Proposition 3.9, we have that $[P(r)]=[P(1)]=[1]=0$. That is, $P(r) \sim r \sim 1$.

If $P(r)=r$ then we are done. Assume $P(r) \neq r$ (hence $r \notin \mathbb{Q}$ ); then $P(r)-r \neq$ 0 . Since $[P(r)]=[r]=0$, we have that $[P(r)-r] \geq 0$. It suffices to show that $[P(r)-r]>0$. Assume to the contrary that $[P(r)-r]=0$. Thus, there is $q \in \mathbb{Q}$ such that $q$ is (strictly) between $r$ and $P(r)$. It follows that either

$$
r<q<P(r)
$$

or

$$
P(r)<q<r
$$

Applying $P$ to the first inequality in (3.2) [resp. the second inequality in (3.3)], we obtain that $P(r)<P(q)=q$ [resp. $P(q)=q<P(r)]$, which contradicts the second inequality in $(3.2)$ [resp. the first inequality in $(3.3)]$. Thus, $[P(r)-r]>0$; and hence $P(r) \approx r$. 
Corollary 3.12. Let $P$ be an order preserving field automorphism of $L$. Then, for all $x \in L^{*},[P(x)]=[x]$ i.e. $P(x) \sim x$.

Proof. By Proposition 3.9, the map $\Gamma: S_{L}=\mathbb{Z} \rightarrow \mathbb{Z}$, given by $\Gamma([x])=$ $[P(x)]$, is an order preserving group automorphism of $\mathbb{Z}=S_{L}$. We need to show that $\Gamma=I$, the identity map on $\mathbb{Z}$. By Proposition 3.9 , we have that

$$
\begin{aligned}
& {[x]=[y] \quad \Rightarrow \quad[P(x)]=[P(y)] \text { and }\left[P^{-1}(x)\right]=\left[P^{-1}(y)\right] ; \text { and }} \\
& {[x]<[y] \quad \Rightarrow \quad[P(x)]<[P(y)] \text { and }\left[P^{-1}(x)\right]<\left[P^{-1}(y)\right] .}
\end{aligned}
$$

We first recall that

$$
\Gamma(0)=\Gamma([1])=[P(1)]=[1]=0 .
$$

Let $d$ be the $L$ number representing the monomial of degree 1 . Since $[d]=1>0=$ [1], we have by Equation (3.5) that

$$
[P(d)]>[P(1)]=[1]=0 \text { and }\left[P^{-1}(d)\right]>\left[P^{-1}(1)\right]=[1]=0 .
$$

Since $d>0$, it follows that $P(d)>0$ and $P^{-1}(d)>0$. If $P(d)=d$, then $[P(d)]=$ $[d]$. If $d<P(d)$, then $[P(d)] \leq[d]$, and hence $0<[P(d)] \leq 1$. Since $[P(d)]$ is an integer, it follows $[P(d)]=1=[d]$. If $P(d)<d$, then $d<P^{-1}(d)$. Thus, $0<\left[P^{-1}(d)\right] \leq[d]=1$ and hence $\left[P^{-1}(d)\right]=1=[d]$. Using Equation (3.4), we then obtain that $\left[P\left(P^{-1}(d)\right)\right]=[P(d)]$, and hence $[P(d)]=[d]=1$. Thus,

$$
\Gamma(1)=1 .
$$

Now let $n \in \mathbb{N}$ be given. Then

$$
\begin{aligned}
\Gamma(n) & =\Gamma\left(\left[d^{n}\right]\right)=\Gamma([\underbrace{d \cdot d \ldots \cdot d}_{n \text { times }}])=\Gamma(\underbrace{[d]+[d]+\cdots+[d]}_{n \text { times }}) \\
& =\underbrace{\Gamma([d])+\Gamma([d])+\cdots+\Gamma([d])}_{n \text { times }}=\underbrace{1+1+\cdots+1}_{n \text { times }} \\
& =n .
\end{aligned}
$$

Finally, let $n \in \mathbb{Z}^{-}$be given; then $-n \in \mathbb{N}$. Since $\Gamma(-n)+\Gamma(n)=\Gamma(-n+n)=$ $\Gamma(0)=0$, it follows that

$$
\Gamma(n)=-\Gamma(-n)=-(-n)=n .
$$

Therefore, $\Gamma(n)=n$ for all $n \in \mathbb{Z}$; and hence $\Gamma=I$.

Example 3.13. Define $P: L \rightarrow L$ as follows: for $x \in L$, write $x=\sum_{k \geq k_{x}} a_{k} d^{k}$ and set $P(x)=\sum_{k \geq k_{x}} 2^{k} a_{k} d^{k}$, where $k_{x}=[x]$. Then $P$ is an order preserving field automorphism of $L$.

Example 3.14. Define $P: \mathcal{R} \rightarrow \mathcal{R}$ as follows: For $x \in \mathcal{R}$, write $\operatorname{supp}(x)=\left\{q_{j}\right.$ : $j \in \mathbb{N}, q_{j_{1}}<q_{j_{2}}$ if $\left.j_{1}<j_{2}\right\}$; then $x=\sum_{j \in \mathbb{N}} a_{j} d^{q_{j}}$ where $a_{j}=x\left[q_{j}\right]$. Set $P(x)=$ $\sum_{j \in \mathbb{N}} a_{j} d^{3 q_{j}}$. Then $P$ is a field automorphism of $\mathcal{R}$, which is order preserving by Lemma 3.7.

Remark 3.15. Note that, in Example 3.14, $P(d)=d^{3} \nsim d$, in contrast with Corollary 3.12 and Example 3.13.

By Lemma 3.7 and Corollary 3.11, we have that $P(r) \approx r$ for any field automorphism $P$ of $\mathcal{R}$ and for any $r \in \mathbb{R}^{*}$. The following example shows that there exist (uncountably many order preserving) field automorphisms of $\mathcal{R}$ which do not preserve every real number. 
ExAmple 3.16. Let $S$ be a transcendence basis for $\mathbb{R} / \mathbb{Q}$; and let $\mathbb{Q}(S)$ denote the field generated by adjoining the elements of $S$ to $\mathbb{Q}$, quipped with the same order as that of $\mathbb{R}$. Then

$S$ is uncountable;

$\mathbb{Q}(S)$ is the smallest field that contains $\mathbb{Q}$ and $S$;

$\mathbb{R}$ is an algebraic extension of $\mathbb{Q}(S)$; and

$\mathbb{R}$ is the real closure of $\mathbb{Q}(S)$. Let

$$
\mathcal{R}_{1}=\left\{x \in \mathcal{R}: x=\sum_{n \in \mathbb{N}} a_{n} d^{q_{n}} \text { with } a_{n} \in \mathbb{Q}(S) \text { for all } n\right\} .
$$

Then $\mathcal{R}_{1}$ is a subfield of $\mathcal{R} ; \mathcal{R}$ is an algebraic extension of $\mathcal{R}_{1}$ and $\mathcal{R}$ is the real closure of $\mathcal{R}_{1}$. We define a map $P_{1}: \mathcal{R}_{1} \rightarrow \mathcal{R}_{1}$ as follows. For all $q \in \mathbb{Q}$, let $P_{1}(q)=q$. Given $r \in \mathbb{Q}(S)$, then there exist $m, n \in \mathbb{N}$ and there exist $r_{1}, \ldots, r_{m}$ and $s_{1}, \ldots, s_{n}$ in $S$ such that $r$ is the quotient of two polynomials $Q_{1}\left(r_{1}, \ldots, r_{m}\right)$ and $Q_{2}\left(s_{1}, \ldots, s_{n}\right)$ with coefficients in $\mathbb{Q}$. That is,

Let

$$
r=\frac{Q_{1}\left(r_{1}, \ldots, r_{m}\right)}{Q_{2}\left(s_{1}, \ldots, s_{n}\right)} .
$$

$$
P_{1}(r)=\frac{Q_{1}\left(r_{1}+d, \ldots, r_{m}+d\right)}{Q_{2}\left(s_{1}+d, \ldots, s_{n}+d\right)} .
$$

Having defined $P_{1}(r)$ for all $r \in \mathbb{Q}(S)$, let now $x \in \mathcal{R}_{1}$ be given. Then

$$
x=\sum_{j \in \mathbb{N}} a_{j} d^{q_{j}} \text { where } a_{j} \in \mathbb{Q}(S) \text { for all } j \in \mathbb{N}
$$

let

$$
P_{1}(x)=\sum_{j \in \mathbb{N}} P_{1}\left(a_{j}\right) d^{q_{j}} .
$$

It follows that $P_{1}$ is an order preserving field automorphism of $\mathcal{R}_{1}$.

Since $\mathcal{R}$ is the real closure of $\mathcal{R}_{1}$, we can extend $P_{1}$ to an order preserving automorphism $P$ of $\mathcal{R}$. Thus,

$$
P(r)=P_{1}(r)=r+d \neq r \text { for all } r \in S \subset \mathbb{R} .
$$

REMARK 3.17. As in Example 3.16, one can in a similar way construct a nontrivial order preserving filed automorphism of any non-Archimedean field extension of $\mathbb{R}$, that does not preserve every real number.

\section{Acknowledgement}

The author would like to acknowledge useful and motivating discussions with Professor Martin Berz of Michigan State University in the early stages of this work.

\section{References}

[1] M. Berz. Calculus and numerics on Levi-Civita fields. In M. Berz, C. Bischof, G. Corliss, and A. Griewank, editors, Computational Differentiation: Techniques, Applications, and Tools, pages 19-35, Philadelphia, 1996. SIAM.

[2] H. Hahn. Über die nichtarchimedischen Größensysteme. Sitzungsbericht der Wiener Akademie der Wissenschaften Abt. 2a, 117:601-655, 1907.

[3] W. Krull. Allgemeine Bewertungstheorie. J. Reine Angew. Math., 167:160-196, 1932.

[4] T. Levi-Civita. Sugli infiniti ed infinitesimi attuali quali elementi analitici. Atti Ist. Veneto di Sc., Lett. ed Art., 7a, 4:1765, 1892. 
[5] T. Levi-Civita. Sui numeri transfiniti. Rend. Acc. Lincei, 5a, 7:91,113, 1898.

[6] S. MacLane. The universality of formal power series fields. Bulletin American Mathematical Society, 45:888, 1939.

[7] S. Priess-Crampe. Angeordnete Strukturen: Gruppen, Körper, projektive Ebenen. Springer, Berlin, 1983.

[8] F. J. Rayner. Algebraically Closed Fields Analogous to Fields of Puiseux Series. J. London Math. Soc., 8:504-506, 1974.

[9] P. Ribenboim. Fields: Algebraically Closed and Others. Manuscripta Mathematica, 75:115150, 1992.

[10] K. Shamseddine. New Elements of Analysis on the Levi-Civita Field. PhD thesis, Michigan State University, East Lansing, Michigan, USA, 1999. also Michigan State University report MSUCL-1147.

[11] K. Shamseddine. On the topological structure of the Levi-Civita field. J. Math. Anal. Appl., 368:281-292, 2010

[12] K. Shamseddine and M. Berz. Exception handling in derivative computation with nonArchimedean calculus. In M. Berz, C. Bischof, G. Corliss, and A. Griewank, editors, Computational Differentiation: Techniques, Applications, and Tools, pages 37-51, Philadelphia, 1996. SIAM.

[13] K. Shamseddine and M. Berz. Convergence on the Levi-Civita field and study of power series. In Proc. Sixth International Conference on p-adic Functional Analysis, pages 283-299, New York, NY, 2000. Marcel Dekker.

[14] K. Shamseddine and M. Berz. The differential algebraic structure of the Levi-Civita field and applications. International Journal of Applied Mathematics, 3:449-465, 2000.

[15] K. Shamseddine and M. Berz. Intermediate values and inverse functions on non-Archimedean fields. International Journal of Mathematics and Mathematical Sciences, 30:165-176, 2002.

[16] K. Shamseddine and M. Berz. Measure theory and integration on the Levi-Civita field. Contemporary Mathematics, 319:369-387, 2003.

[17] K. Shamseddine and M. Berz. Analytical properties of power series on Levi-Civita fields. Annales Mathématiques Blaise Pascal, 12(2):309-329, 2005.

[18] K. Shamseddine and M. Berz. Generalized power series on a non-Archimedean field. Indagationes Mathematicae, 17(3):457-477, 2006.

[19] K. Shamseddine and M. Berz. Intermediate value theorem for analytic functions on a LeviCivita field. Bulletin of the Belgian Mathematical Society- Simon Stevin, 14:1001-1015, 2007.

[20] K. Shamseddine and V. Zeidan. One-dimensional optimization on non-Archimedean fields. Journal of Nonlinear and Convex Analysis, 2:351-361, 2001.

[21] K. Shamseddine and V. Zeidan. Constrained second order optimization on non-Archimedean fields. Indagationes Mathematicae, 14:81-101, 2003.

Department of Physics and Astronomy, University of Manitoba, Winnipeg, ManiTOBA R3T 2N2, CANADA

E-mail address: khodr@physics.umanitoba.ca 